\title{
Remote Sensing and Geospatial Analytics for Sustainable Development Assessment in Urban Areas
}

\author{
Xiaojun Yang ${ }^{\text {a,c*}}$, Di Shi ${ }^{b}$, Feilin Lai ${ }^{\mathrm{a}}$, Shijun Jiang ${ }^{\mathrm{c}}$, Ranhao Sun ${ }^{\mathrm{d}}$ \\ ${ }^{a}$ Department of Geography, Florida State University, Tallahassee, Florida 32306, U.S.A., xyang@fsu.edu \\ ${ }^{b}$ Department of Geography and Atmospheric Science, University of Kansas, Lawrence, KS 66045-7613, U.S.A. \\ ${ }^{c}$ Institute of Groundwater and Earth Sciences, Jinan University, Guangzhou, Guangdong 510632, P. R. China \\ ${ }^{d}$ Research Center for Eco-Environmental Sciences, Chinese Academy of Sciences, Beijing 100085, P.R. China \\ * Corresponding author
}

Keywords: Satellite remote sensing, geospatial analytics, sustainable development, ecological sustainability, cities

\begin{abstract}
:
With the concern over the rising global population, the diminishing natural resources, and the continued environmental degradation, the demand for sustainable development has become increasingly high. There is an urgent need to conduct research that can help assess the progress towards various sustainable development goals, such as the one formulated by the United Nations in 2015, which is arguably the most comprehensive scheme. In this paper, we review a set of techniques and methods that can be used for sustainable development assessment, including place-focused studies, observations and monitoring, analytic methods, and models. While scholars from various disciplines contribute to the sustainability research using different methods, we herewith highlight the role of the spatially explicit approach that has its root in the field of geography but has been increasingly adopted in sustainability science. We finally present a case study targeting all the major cities in China, which is based on the use of satellite remote sensing and geospatial analytical methods to derive indicators for assessing sustainable development goals targeting the terrestrial and environmental sustainable development process. We conclude that synthesizing remote-sensing derived measurements with data from other sources such as in situ surveys and social censuses and combining quantitative analyses with other approaches can help better assess and monitor the progress towards achieving sustainable development goals.
\end{abstract}

